\title{
URBAN INFRASTRUCTURE MONITORING WITH A SPATIALLY ADAPTIVE MULTI-LOOKING INSAR TECHNIQUE
}

\author{
Jayanti Sharma $^{(1)}$, Jayson Eppler ${ }^{(1)}$, Jennifer Busler ${ }^{(1)}$ \\ (1) MDA Systems Ltd., 13800 Commerce Parkway, Richmond, British Columbia, Canada, V6V2J3, \\ Email:jasharma@mdacorporation.com
}

\begin{abstract}
Surface displacements for urban infrastructure monitoring are derived using Interferometric Synthetic Aperture Radar (InSAR). The analysis uses a novel InSAR method, Homogenous Distributed Scatterer (HDS)-InSAR, that exploits both persistent point and coherent distributed scatterers using adaptive multilooking of statistically homogenous pixel neighbourhoods. An unwrapped phase model incorporating meteorological data enables separation of temperature-correlated displacement from potentially hazardous long-term trends. Results are presented over the Canadian cities of Regina, Winnipeg and Montreal using RADARSAT-2 and TerraSAR-X data. The new combination of HDS-InSAR and the extended phase model permits large areas of infrastructure to be remotely monitored on a regular basis and enables a more targeted monitoring process to help identify infrastructure at greatest risk for damage.
\end{abstract}

\section{INTRODUCTION}

Many cities have aging infrastructure where, due to limited resources, not all infrastructure can be surveyed on a regular basis using conventional methods. Multitemporal Interferometric Synthetic Aperture Radar (InSAR) techniques allow simultaneous high accuracy, fine spatial resolution and broad spatial coverage measurements of surface deformation. Mapping of urban surface displacement with InSAR can help identify areas where the durability and stability of infrastructure such as buildings, bridges, tarmac, roads and underground pipes are threatened.

The goal of this work is to apply advanced InSAR techniques to detect, quantify, and visualize subtle surface displacement of urban infrastructure. Section 2 reviews the InSAR processing chain used to derive deformation results. Section 3 describes the data and test sites used in the analysis and Section 4 presents results over several Canadian cities derived from RADARSAT-2 and TerraSAR-X image stacks. A summary and discussion of further improvements to the methods is given in Section 5.

\section{METHOD}

\subsection{Spatially adaptive multi-looking}

For urban infrastructure monitoring, it is desired to use a high-resolution InSAR technique capable of capturing detailed infrastructure from both point targets such as metal and building corners, and coherent distributed targets such as asphalt and concrete surfaces. In this work we employ the Homogeneous Distributed Scatterer (HDS)-InSAR [1] technique inspired by a new class of advanced InSAR methods combining the advantages of Differential InSAR (DInSAR) and Persistent Scatterer Interferometry (PSI) [2][3].

HDS uses spatially adaptive multi-looking over local neighbourhoods with statistically similar amplitude distributions [2] to suppress noise over distributed targets while preserving point targets. By averaging a pixel only with neighbours presenting similar scattering properties, the boundaries between areas representing different types of backscatter are preserved. The adaptive filtering reduces the noise of smooth, lowbackscatter areas such as asphalt and bare ground, while optimally preserving the spatial resolution to increase both the accuracy and geographic precision of the deformation result. The HDS method requires roughly the same number of scenes as PSI, typically 15 scenes or more for robust deformation results, while increasing point densities by an order of magnitude over PSI.

\subsection{Separation of linear and seasonal displacement}

Of particular importance in urban monitoring is the separation of temperature-correlated displacement that is anticipated in infrastructure design from long-term displacement trends which may pose a hazard to infrastructure. To achieve this separation a linear phase model of the unwrapped data is used to simultaneously estimate residual height error, linear deformation rate, and temperature-correlated displacement [4]. Regional temperatures [5] are input to the model to determine the thermal dilation coefficient for each HDS target. If temperature-correlated displacement is not taken into account, linear deformation rate estimates may be biased, where this bias can be considerable for short observation periods of less than a few years [4]. 
If seasonal deformation is due to precipitation, soil moisture, or other combination of factors, but still displays significant correlation with temperature, seasonal and long-term linear displacement can still be approximately separated using this model.

\subsection{InSAR processing chain}

The InSAR processing chain used to derive surface deformation from a network of interferograms is presented in Fig.1. The new steps specific to HDS (spatially adaptive multi-looking) are in orange and to the unwrapped phase model are in green.

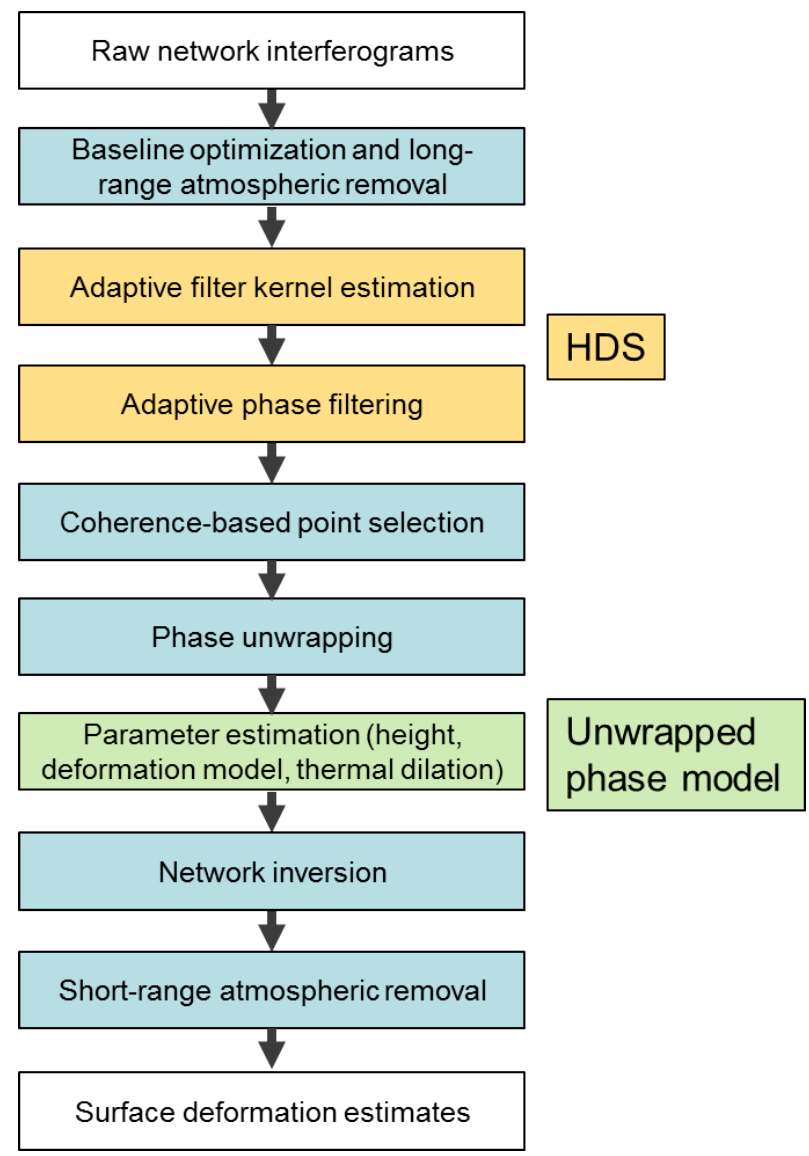

Figure 1, InSAR Processing Chain.

\section{DATA AND TEST SITES}

\subsection{SAR and ancillary data}

Medium-to-high resolution RADARSAT-2 image stacks were acquired over the Canadian cities of Winnipeg, Regina, and Montreal, and a TerraSAR-X Stripmap stack over Montreal to capture representative examples of urban infrastructure including buildings, bridges, tarmac, and roads.

A summary of the characteristics of the SAR image stacks used in this analysis is given in Tab.1. The RADARSAT-2 Multi-Look Fine data allows for wide area analysis of large urban footprints while the Spotlight mode is used to image smaller areas at a finer spatial resolution.

Ancillary data used in the analysis includes historical temperature and precipitation information from Environment Canada [5] and digital elevation models (DEMs) from the Shuttle Radar Topography Mission (SRTM) [6]. A high resolution (5 m posting) LiDAR DEM over a subset of the Regina footprint was also available courtesy of the City of the Regina.

\subsection{Test sites}

Regina is representative for smaller cities with expansive semi-urban terrain where building structure is less dense and population density is lower. Regina is built on expansive clay soils that expand when wetted and contract as they dry, causing surface displacement. Severe stress is placed on infrastructure leading to the cracking of pavement and building foundations, and other issues including pipe and water main breakages. One RADARSAT-2 spotlight stack is analyzed at this site.

Winnipeg lies at the confluence of the Red and the Assiniboine rivers, and is prone to flooding in the spring that can cause significant structural damage. RADARSAT-2 Multi-Look Fine and Spotlight data were acquired over Winnipeg, and we examine the trade-off between high spatial resolution and increased spatial coverage.

Table 1, Summary of InSAR Stacks from RADARSAT-2 (RS-2) and TerraSAR-X (TSX)

\begin{tabular}{llllllllll} 
Site & Sensor & Beam & Pass & $\begin{array}{l}\text { Incidence } \\
\text { Angle }\left[{ }^{\circ}\right]\end{array}$ & $\begin{array}{l}\text { Ground } \\
\text { res }[\mathbf{m}]\end{array}$ & $\begin{array}{l}\text { Azimuth } \\
\text { res }[\mathbf{m}]\end{array}$ & $\begin{array}{l}\text { Swath } \\
{[\mathbf{k m}]}\end{array}$ & Scenes & Start/End dates \\
\hline \hline \multirow{2}{*}{ Regina } & RS-2 & SLA1 & Asc & 30.5 & 2.8 & 0.8 & $18 \times 8$ & 23 & $2011 / 06-2013 / 03$ \\
Winnipeg & RS-2 & MF3 & Asc & 20.6 & 4.5 & 4.6 & $50 \times 50$ & 21 & $2011 / 07-2012 / 12$ \\
& RS-2 & SLA70 & Asc & 42.8 & 4.6 & 0.8 & $18 \times 8$ & 20 & $2011 / 07-2012 / 11$ \\
Montreal & RS-2 & SLA26 & Asc & 48.4 & 2.1 & 0.8 & $18 \times 8$ & 18 & $2011 / 02-2013 / 03$ \\
& TSX & SM_011 & Asc & 39.6 & 1.9 & 3.3 & $30 \times 50$ & 40 & $2011 / 09-2013 / 05$
\end{tabular}


Montreal is the second largest city in Canada with aging bridges, highways, and overpasses that can be monitored for displacement hazards. There are a number of historic buildings in the downtown and old port area, as well as several unusual structures such as the geodesic dome of the Montreal Biosphere to examine with InSAR. One RADARSAT-2 (Cband) Spotlight stack and one TerraSAR-X (Xband) Stripmap stack were acquired over similar time periods, enabling a comparison of the $\mathrm{C} / \mathrm{X}$-band inverted deformations.

\section{RESULTS}

\subsection{Regina}

Applying HDS-InSAR and the unwrapped phase model described in Section 2, surface deformations were inverted for the Regina RADARSAT-2 Spotlight stack. The seasonal displacement rate map measured in the radar line-of-sight (LOS) direction is shown in Fig.2. Areas where the surface moves towards the radar during colder temperatures are shown in yellow and red, whereas green areas show negligible seasonal displacement. The average radar intensity image over the entire image stack is shown in the background. All images are geocoded to North-up.

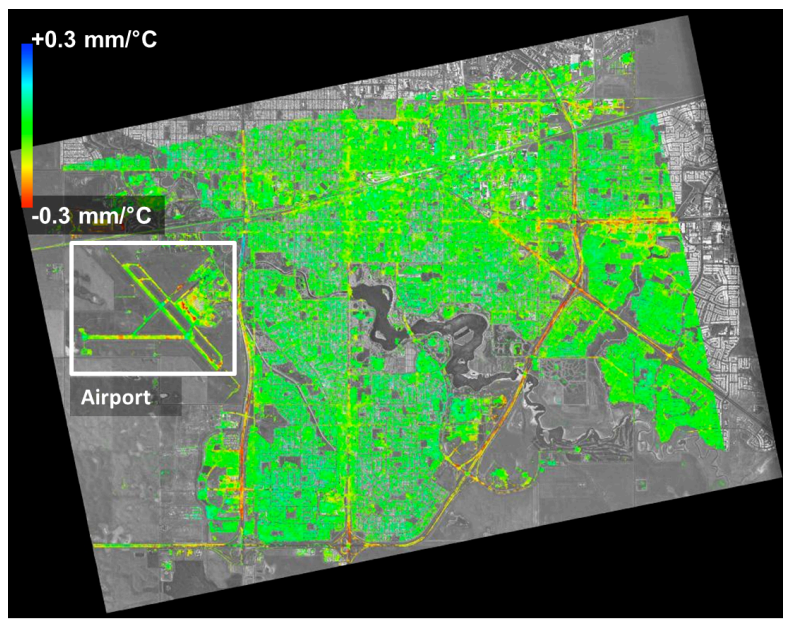

RADARSAT-2 Data and Products $\odot$ MacDonald, Dettwiler and Associates Ltd. (2011-2013). All Rights Reserved. RADARSAT is an official mark of the Canadian Space Agency.

Figure 2, Temperature-correlated displacement in the radar LOS direction for Regina, RADARSAT-2.

Although long-term linear displacement and temperature-correlated displacement are benign across most of the city, some areas such as the airport tarmac and several major roads (see Fig.2) experience strong seasonal displacement consistent with frost heave that can result in significant damage to asphalt.

An enlarged subset of the Regina airport from Fig.2 is shown in Fig.3. Areal coverage of the airport tarmac and buildings is dense, yielding information on both distributed and point targets with the HDS method. The airport tarmac shows minimal linear deformation trends,
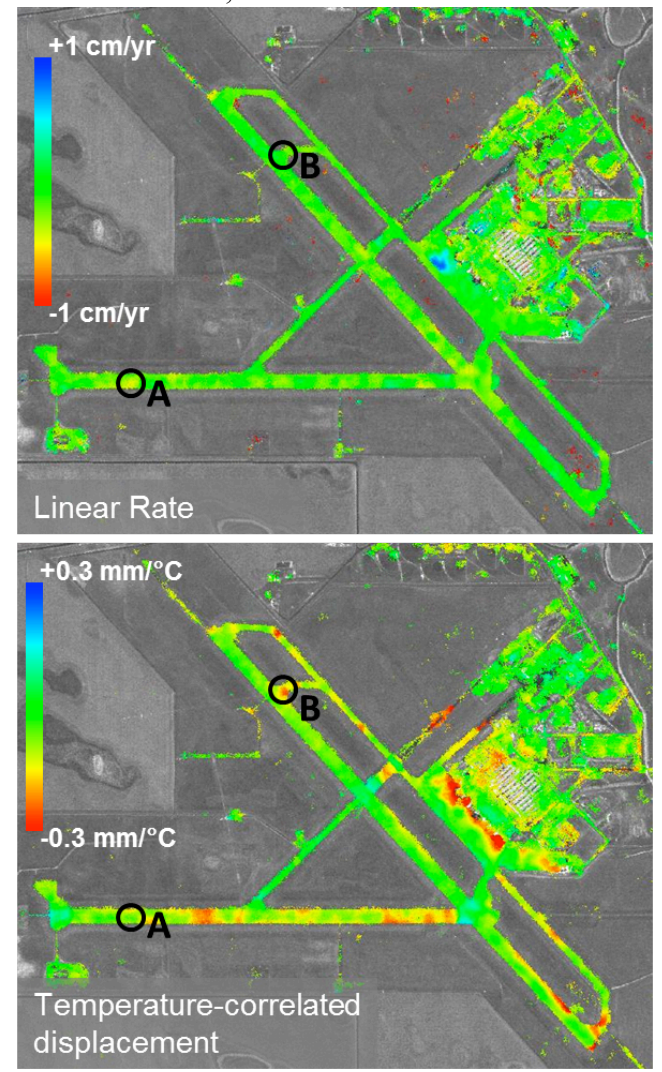

RADARSAT-2 Data and Products ( $)$ MacDonald, Dettwiler and Associates Ltd. (2011-2013). All Rights Reserved. RADARSAT is an official mark of the Canadian Space Agency.

Figure 3, Linear displacement rate (top) and temperature-correlated displacement (bottom) in the radar LOS for Regina airport subset (see Fig.2), derived from RADARSAT-2. Deformation time series for points $(A)$ and $(B)$ are displayed in Fig. 4.

although seasonal motion with amplitudes of up to 1 $\mathrm{cm}$ per year is detected. The motion is consistent with winter frost heave resulting when water in the wet clay soils underlying Regina freezes to form ice lenses, causing uplift.

Deformation time series for two points in Fig.3 are shown in Fig.4. Both 'raw' deformation (blue points) and temporally filtered deformation time series (blue solid lines) [7] are plotted. Point (A) shows a slight subsidence trend $(\sim 0.25 \mathrm{~cm} /$ year $)$ superimposed on a weak temperature-correlated displacement signal. Point (B) has a negligible linear rate but displays strong seasonal displacement. Mean daily temperatures on acquisition dates (in magenta) are overlaid on the point (B) time series showing that the coldest periods in January coincide with the strongest movement towards the radar, consistent with frost heave. 
Although seasonal and long-term linear displacement have been separated through correlation with mean daily air temperature, this does not mean that deformation is solely caused by temperature
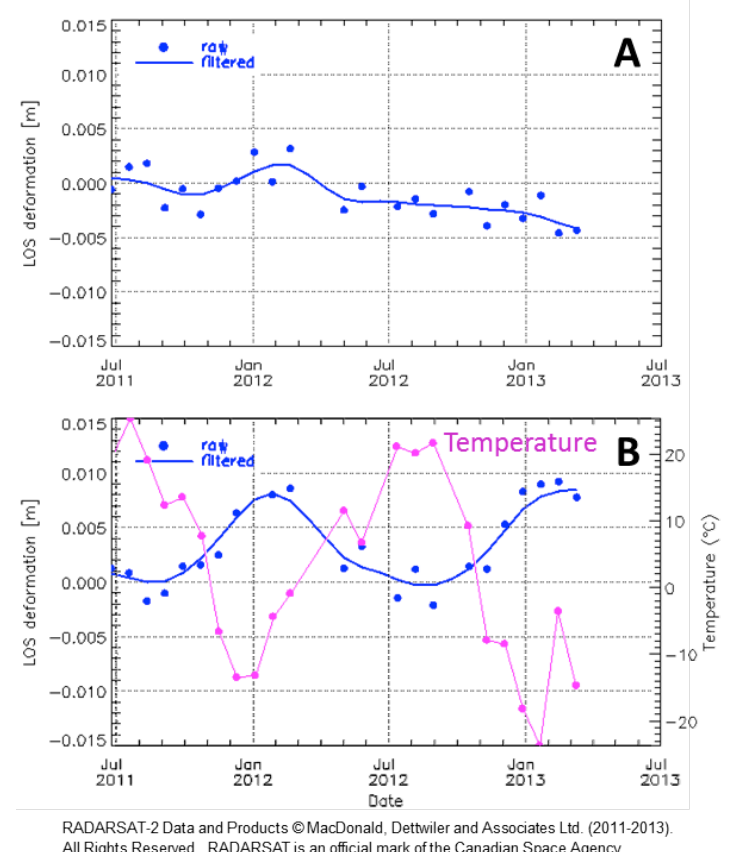

Figure 4, RADARSAT-2 LOS deformation time series for points ( $A$ ) (top) and (B) (bottom) from Fig.3.

Both raw (blue points) and temporally filtered deformations (blue lines) are plotted. Bottom image also plots mean daily temperature (magenta) on image acquisition days referenced to the right hand $y$ axis.

fluctuations. Seasonal displacement is likely due to a complex interplay of temperature, precipitation, snow distribution and soil moisture, but which correlates reasonably well with temperature.

\subsection{Winnipeg}

Surface deformations inverted from the two RADARSAT-2 stacks over Winnipeg display less seasonal displacement compared to Regina and minimal linear displacement rates. However, persistent displacement was identified over several residential areas. Fig. 5 shows a subsiding residence in the older Winnipeg community of Burrows, where linear displacement rates from the Spotlight stack are overlaid on Google Earth optical imagery. Cracks in the residence are clearly visible in a Google Earth Street View image of the house taken April 2012, midway through the RADARSAT-2 observation period, indicating long-term structural damage. Deformation time series indicate subsidence rates of up to $2 \mathrm{~cm} /$ year. This deformation was not clearly resolved with the Multi-Look Fine stack.

A second example is strong surface displacement of the new Rivergrove residential area in Winnipeg. The residential area was captured in both the Spotlight (SLA70) and Multi-Look Fine (MF3) stacks, where Fig.6 displays a comparison of the vertical cumulative

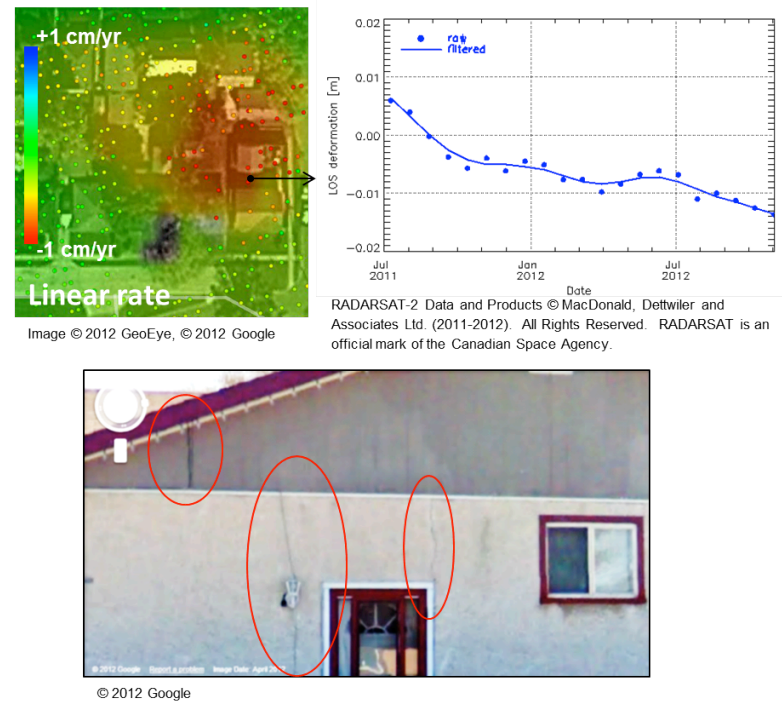

Figure 5, Top: LOS linear rate map ( $R A D A R S A T-2$,

$S L A 70$ stack, colour saturation at $1 \mathrm{~cm} /$ year) of a subsiding older Winnipeg residence overlaid on optical imagery and accompanying deformation time series.

Bottom: cracks visible in the residence are circled in red (image date: April 2012).

deformation between July 2011 and November 2012 . This assumes displacement primarily in the vertical direction in order to compare results from the two significantly different incidence angles (see Tab.1). The deformation in Fig.6 is consistent with a slumping of the

riverbank (subsidence combined with horizontal movement towards the river), with the deformation becoming stronger in the vicinity of the river.

The observed displacement pattern in Fig.6 is generally consistent between SLA70 and MF3, although the higher-resolution Spotlight data has denser point coverage including much better road coverage. However, the Multi-Look Fine stack successfully captures the general displacement pattern and offers 10 times the areal coverage compared to Spotlight.

\subsection{Montreal}

Long-term linear displacement is benign over most of Montreal for both the RADARSAT-2 and TerraSAR-X stacks, although several areas of the 
city experience temperature-correlated displacement including bridges, quays, and steel infrastructure. Fig.7 displays the LOS linear rate and temperaturecorrelated displacement for the RADARSAT-2 Spotlight stack in the historic Old Port area of Montreal. Several of the piers and promenades are experiencing persistent deformation, identified by their strong linear displacement rates. Deterioration and ongoing repair of the piers has been documented in annual reports by the Old Port of Montréal Corporation [8].
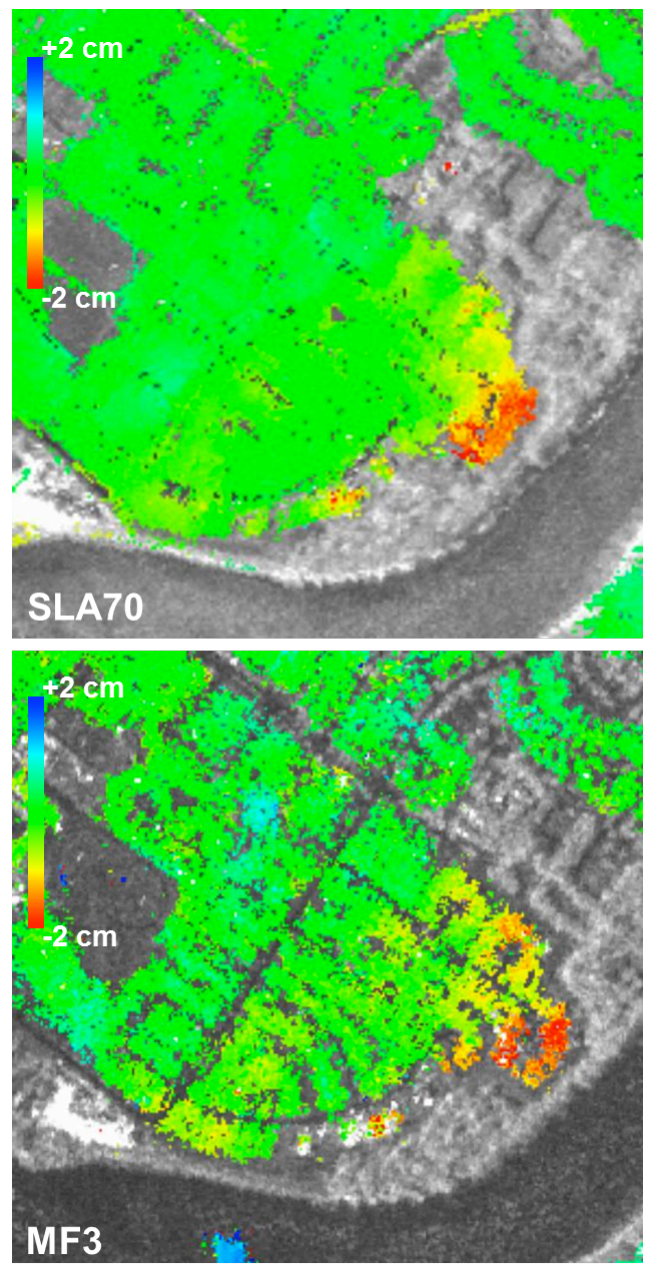

RADARSAT-2 Data and Products $\odot$ MacDonald, Dettwiler and Associates Ltd. (2011-2012). All Rights Reserved. RADARSAT is an official mark of the Canadian Space Agency.

Figure 6, Cumulative vertical deformation over Winnipeg residential area spanning Jul-2011 to Nov2012 for the RADARSAT-2 SLA70 stack (top) and MF3 stack (bottom).

Linear rate and temperature-correlated displacement are also inverted for the TerraSAR-X Stripmap stack, shown in Fig.8. An incidence angle difference of $9^{\circ}$ prevents a direct comparison of RADARSAT-2 and TerraSAR-X LOS displacements, but trends are consistent between the two ascending stacks. The RADARSAT-2 result has slightly higher target densities than TerraSAR-X, particularly over roads. This may be partially due to the higher temporal coherence and thus higher phase quality of the Cband compared to the $\mathrm{X}$-band data through the winter acquisitions. However, a second contributing factor is that the RADARSAT-2 Spotlight data have a higher azimuth resolution and were multi-looked by a factor of 4 in azimuth to obtain roughly square pixels; this reduces the phase noise compared to the single-look TerraSAR-X Stripmap data.

In Fig.7 and Fig.8 linear rate and temperaturecorrelated

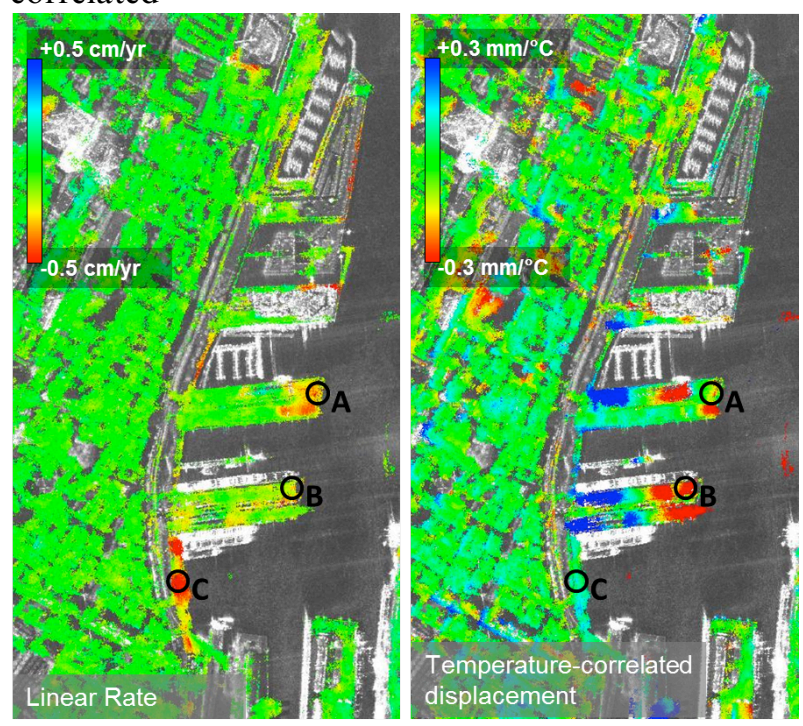

RADARSAT-2 Data and Products $\mathbb{O}$ MacDonald, Dettwiler and Associates Ltd. (2011-2013). All Rights Reserved. RADARSAT is an official mark of the Canadian Space Agency.

Figure 7, Linear displacement rate (left) and temperature-correlated displacement (right) in the radar LOS for Montreal's Old Port derived from RADARSAT-2. Deformation time series for points (A), (B) and (C) are displayed in Fig.9. 

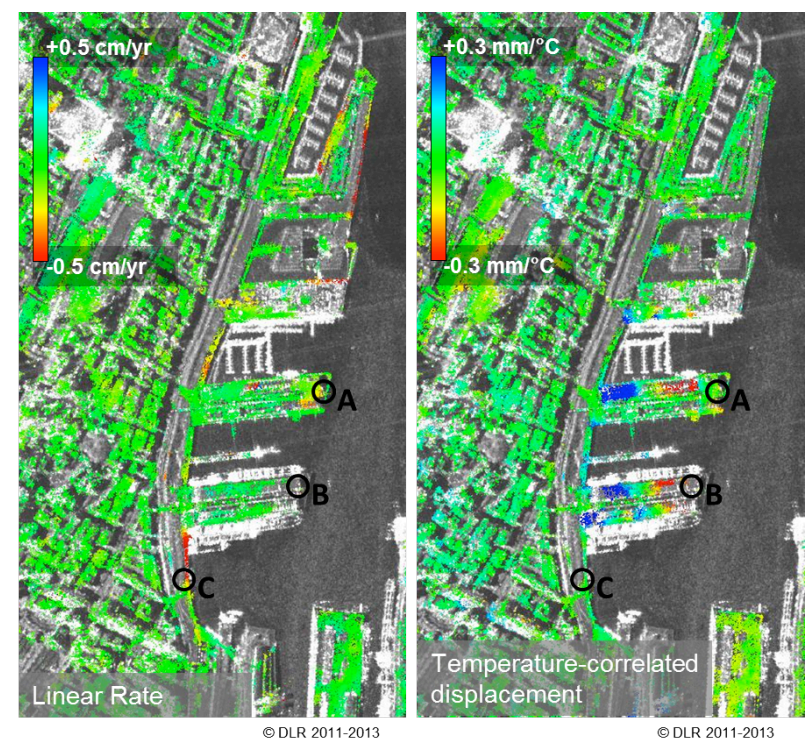

Figure 8, Linear displacement rate (left) and temperature-correlated displacement (right) in the radar LOS for Montreal's Old Port derived from

TerraSAR-X. Deformation time series for points (A), $(B)$ and $(C)$ are displayed in Fig.9.

deformation maps are presented in the LOS direction without assumption of the direction of deformation. To compare deformation time series between the two sensors, displacements are projected to the horizontal (roughly East-West direction), assuming most temperature-correlated displacement and a sizable portion of long-term linear displacement is horizontal, for instance slumping towards the water. However, the
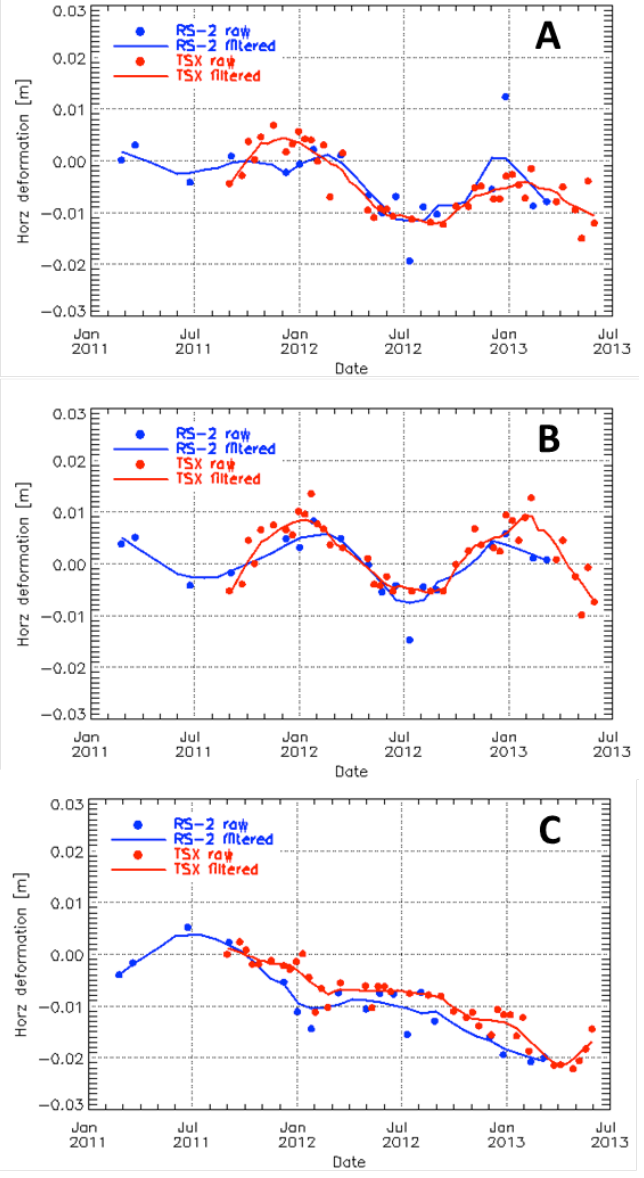

RADARSAT-2 Data and Products $\odot$ MacDonald, Dettwiler and Associates Ltd. (2011-2013). All Rights Reserved. RADARSAT is an official mark of the Canadian Space Agency.

Figure 9, Deformation time series projected to the horizontal for points $(A),(B)$ and $(C)$ from Fig. 7 and

Fig.8. RADARSAT-2 displacements are in blue and TerrSAR-X displacements are in red.

presence of any subsidence or uplift will introduce errors in the comparison. Deformation time series are arbitrarily zeroed at October $1^{\text {st }}, 2011$ in order to compare sensor stacks. Time series for points (A), (B) and (C) from Fig.7 and Fig.8 are presented in Fig.9.

Point (A) is located on the deteriorating King Edward Pier and displays slow long-term displacement of $\sim 0.5$ $\mathrm{cm} /$ year superimposed on a stronger seasonal displacement signal. In Fig.7 and Fig.8 HDS-InSAR has separated long-term displacement trends from the seasonal effects. While thermal dilation has likely been accounted for in the pier's design, the long-term displacement trend could eventually lead to hazardous conditions and may be of interest to engineers and urban planners for early detection of infrastructure damage. Point (B) on Alexandra Quay illustrates strong seasonal displacement and Point (C) on the promenade has a high linear rate of $\sim 1 \mathrm{~cm} /$ year away from the radar consistent 
with slumping towards the river.

A canonical example of the strong thermal dilation and contraction of steel structures is provided by the Montreal Biosphere. The Biosphere (formerly the US pavilion for Expo 67) consists of an enclosed structure of steel and acrylic cells. Steel responds to higher temperatures by expanding and to colder temperatures by contracting. Fig. 10 is a photograph of the Biosphere museum and Fig.11 and Fig.12 display LOS deformation maps inverted from the RADARSAT-2 and TerraSAR-X stacks, respectively.

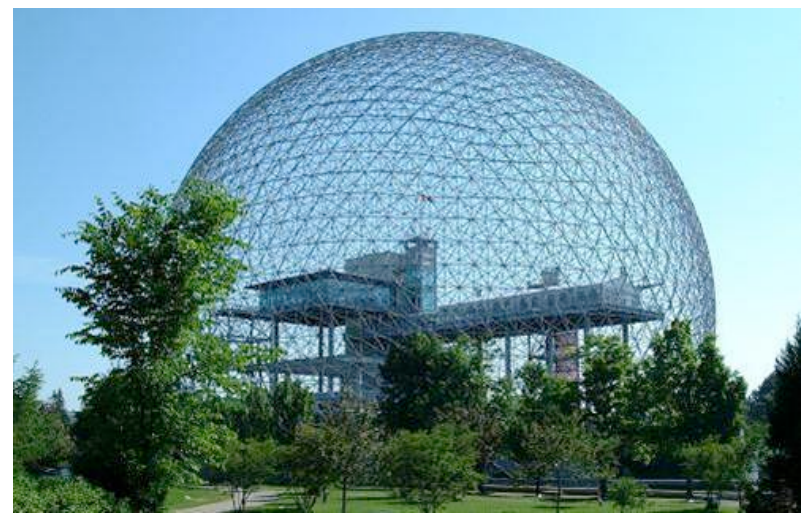

Figure 10, Montreal Biosphere environmental museum, $76 \mathrm{~m}$ in diameter and $63 \mathrm{~m}$ high [9]. (Source of image: Environment Canada, http://www.ec.gc.calbiosphere.)

Displacement rates derived from the two sensors are consistent and target densities comparable over most structures except the geodesic dome, where RADARSAT-2 is somewhat denser. The footbridge to the South-East of the Biosphere also displays strong temperature-correlated displacement in both stacks, and likely has expansion joints on both ends. Temperature-correlated displacement has been separated from long-term linear displacement, where the Biosphere and nearby structures are all stable showing no long-term displacement trends.

\section{SUMMARY}

In this work RADARSAT-2 and TerraSAR-X data were successfully used to map deformation over the Canadian cities of Regina, Winnipeg, and Montreal. HDSInSAR, a spatially adaptive multi-looking technique, was used to identify areas where the durability and stability of a variety of infrastructure (residential areas, airports, roads, and piers) are affected by surface displacement. In combination with HDS, an unwrapped phase model is employed to simultaneously estimate residual height error, linear deformation rate, and temperature-correlated displacement.
The HDS-InSAR results are promising, particularly using high-resolution Spotlight data to detect seasonal deformation and potentially hazardous long-term deformation trends of urban infrastructure at high accuracies and dense spatial coverage. Of particular
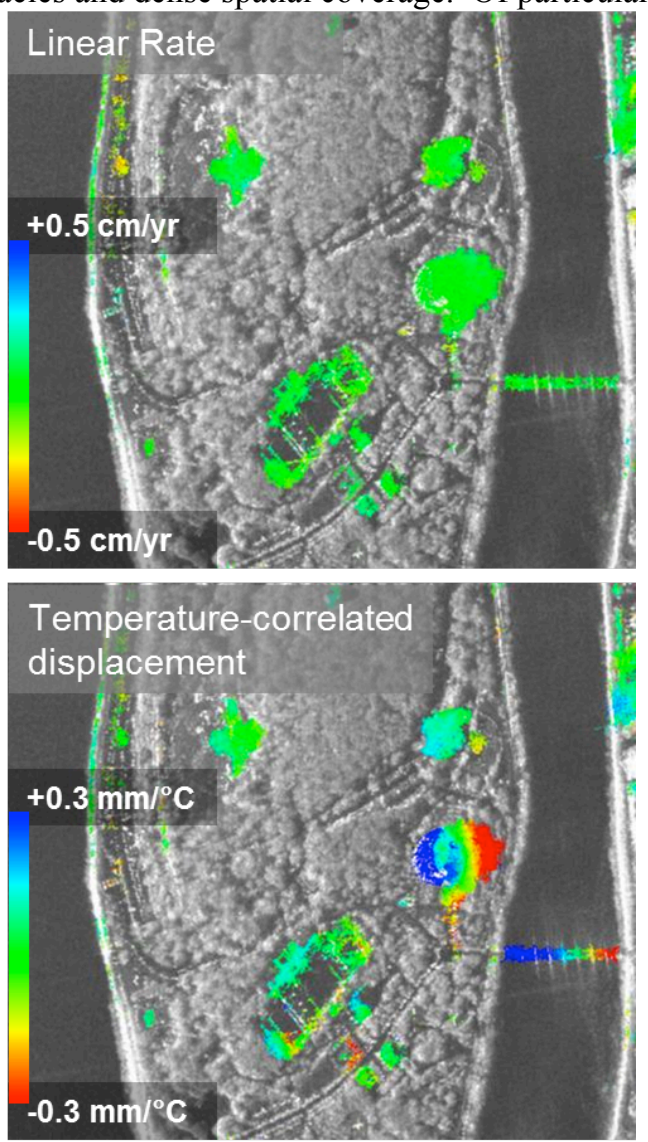

RADARSAT-2 Data and Products (C) MacDonald, Dettwiler and Associates Ltd. (2011-2013). All Rights Reserved. RADARSAT is an official mark of the Canadian Space Agency.

Figure 11, Linear displacement rate (top) and temperature-correlated displacement (bottom) in the radar LOS for the Montreal Biosphere (centre-right circular structure) derived from RADARSAT-2.

importance is the separation of temperature-correlated displacement (estimated using meteorological data and the unwrapped phase model) that is anticipated in infrastructure design from long-term displacement trends which may pose a threat to infrastructure.

Due to limited resources not all infrastructure can be surveyed on a regular basis using conventional methods. It is envisioned that HDS-InSAR combined with an extended phase model would allow wide areas of infrastructure to be routinely and remotely monitored, identifying infrastructure at greatest risk for damage.

Further improvement to the presented results would be 
anticipated using (i) multiple seasons of data for enhanced separation of seasonal and long-term trends, (ii) more localized temperature measurements on infrastructure of interest since buildings and bridges can display significant temperature gradients due to sun, shading, and elevation, and (iii) soil moisture estimates in the extended phase model to localize regions at risk of frost heave and water main breaks.
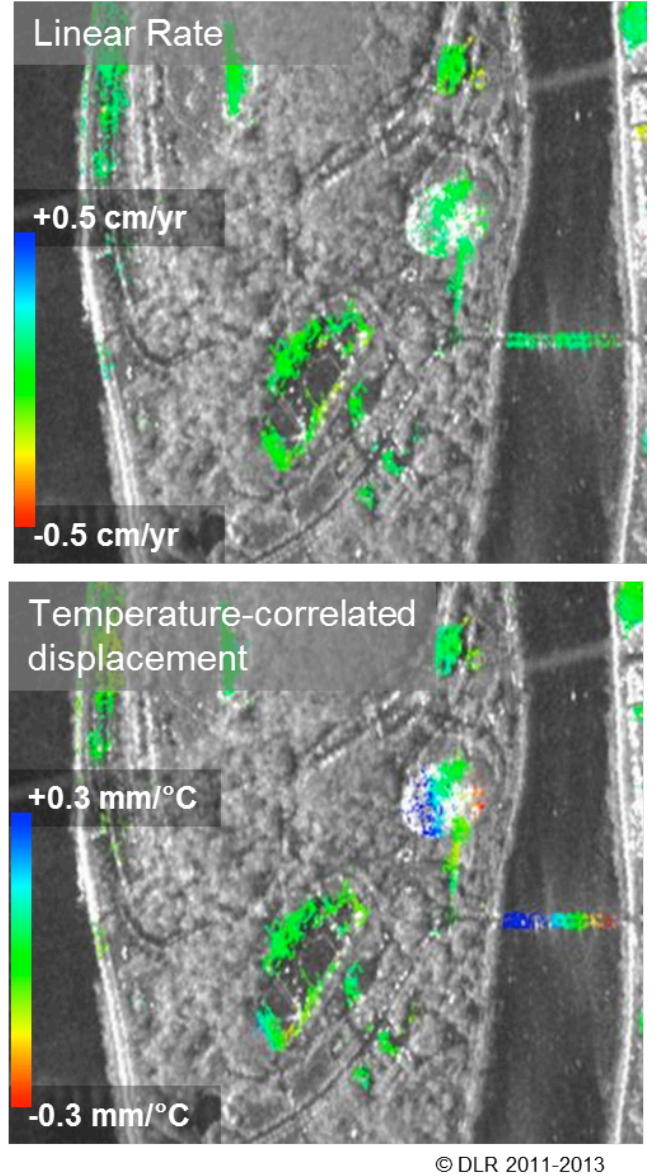

Figure 12, Linear displacement rate (top) and temperature-correlated displacement (bottom) in the radar LOS for the Montreal Biosphere (centre-right circular structure) derived from TerraSAR-X.

\section{ACKNOWLEDGEMENTS}

This work has been partially funded by the Canadian Space Agency (CSA) under the Earth Observation Application Development Program (EOADP) contract \#9F028-090155. The authors thank the Natural Resources Canada Centre for Sustainable Infrastructure Research (NRC CSIR) for their collaboration on this project. RADARSAT-2 images were provided by the CSA and TerraSAR-X images by the German Aerospace Center (DLR) under proposal ID GEO1027.

\section{REFERENCES}

1. Rabus, B., Eppler, J., Sharma, J., \& Busler, J. (2012).
Tunnel monitoring with an advanced InSAR technique. Proceedings of SPIE., 8361, 83611F$83611 \mathrm{~F}-10$.

2. Parizzi, A. \& Brcic, R. (2011). Adaptive InSAR Stack Multilooking Exploiting Amplitude Statistics: A Comparison Between Different Techniques and Practical Results. IEEE Geosci. Remote Sens. Letters, 8(3), 441-445.

3. Ferretti, A., Fumagalli, A., Prati, C., Rocca, F., \& Rucci, A. (2011). A new algorithm for processing interferometric data-stacks: SqueeSAR. IEEE Trans. Geosci. Remote Sens., 49(9):3460-3470.

4. Monserrat, O., Crosetto, M., Cuevas, M. \& Crippa, B. (2011). The Thermal Expansion Component of Persistent Scatterer Interferometry Observations. IEEE Geosci. Remote Sens. Letters, 8(5), 864-868.

5. "National Climate Data and Information Archive". Environment Canada. Retrieved from http://www.climate.weatheroffice.gc.ca on April 15, 2013.

6. "SRTM 90m Digital Elevation Database v4.1", CGIAR CSI Consortium for Spatial Information. Retrieved from http://www.cgiarcsi.org/data/srtm-90m-digital-elevationdatabase-v4-1 on February 26, 2015.

7. Cleveland, W.S. (1979). Robust Locally Weighted Regression and Smoothing Scatterplots, Journal of the American Statistical Association, 74(368),829-836.

8. Old Port of Montréal Corporation, Annual Report (2008-2009). Retrieved from http://www.oldportcorporation.com/documents /corpo/pdf/OldPort-Annual-Report-2008-09.pdf on March 8, 2015.

9. CADDET Energy Efficiency. (1998). "Geothermal heating and cooling for Montreal's Biosphere", Retrieved from http://www.nrcan.gc.ca/sites/oee.nrcan.gc.ca/file s/pdf/publications/infosource/pub/ici/caddet/eng lish/pdf/R299.pdf on March 8, 2015. 